\title{
Geração Automática de Receitas Culinárias para Pessoas com Restrições Alimentares
}

\author{
Larissa F. S. Britto ${ }^{1}$, Luciano D. S. Pacífico ${ }^{1}$, Teresa B. Ludermir ${ }^{2}$ \\ ${ }^{1}$ Departamento de Computação (DC) \\ Universidade Federal Rural de Pernambuco (UFRPE) - Recife, PE - Brasil \\ ${ }^{2}$ Centro de Informática - CIn \\ Universidade Federal de Pernambuco - UFPE - Recife, PE - Brasil \\ \{larissa.feliciana, luciano.pacifico\}@ufrpe.br, tbl@cin.ufpe.br
}

\begin{abstract}
Even with the increasing number of web pages dedicated to sharing culinary recipes, users may still have difficulty to find specific dishes due to the massive amount of data available in such repositories. These websites have resorted to recommendation systems to make the process of finding the ideal recipe easy to the users. However, the problem persists for people who have dietary restrictions or food intolerances, due to the reduced number of recipes aimed to such public. In this work we propose an automatic recipe generation approach, based on the substitution of ingredients using linear classifiers, in an attempt to help these users finding recipes that contemplate both their desires and food restrictions. An experimental evaluation was performed, adapting already existing recipes as diabetic friendly and dairy-free recipes. The experimental results showed that the proposed method was able to find adequate ingredient substitutes for most of the evaluated scenarios.
\end{abstract}

Resumo. Mesmo como o aumento no número de páginas web dedicadas ao compartilhamento de receitas culinárias, usuários ainda podem encontrar dificuldade na busca por pratos específicos devido à enorme quantidade de dados contidos nos repositórios. Esses websites têm recorrido a sistemas de recomendação para facilitar o processo de busca. Porém, para pessoas que possuem restrições alimentares ou intolerâncias, o problema persiste, devido à pequena porcentagem de receitas destinadas a esse público. Neste trabalho propomos um método de geração automática de receitas, baseado na substituição de ingredientes utilizando classificadores lineares, numa tentativa de ajudar os usuários a encontrarem receitas que contemplem suas vontades e restrições alimentares. Uma análise experimental foi realizada, adaptando receitas para dietas diabéticas e intolerantes à lactose. Os resultados apontam que o método obteve substitutos adequados na grande maioria dos cenários testados.

\section{Introdução}

A alimentação desempenha um papel fundamental para os seres humanos, seja por questões biológicas (a alimentação provê energia, auxilia no reparo das perdas sofridas pelo organismo, atua no combate e prevenção de doenças, funcionando ainda como agente regulador do corpo), como também por se caracterizar como um forte elemento sociocultural. A tomada de decisão em relação ao tipo de alimento que se deseja consumir a cada 
momento na rotina diária de uma pessoa é um processo complexo, que envolve várias escolhas relacionadas a fatores como a quantidade de tempo disponível, local no qual deseja-se realizar a refeição, fatores culturais e sociais, disponibilidade dos alimentos, valor nutricional da comida, e gostos pessoais.

Com o advento das Tecnologias de Informação e Comunicação (TICs), a quantidade de dados disponíveis a um usuário comum tem aumentado exponencialmente. De forma semelhante, é cada vez mais fácil o acesso a uma grande variedade de tipos de alimentos das mais diversas origens, seja pela busca por receitas e ingredientes na Internet ou em mercados locais, seja pela interação com indivíduos e restaurantes provenientes das mais variadas etnias, raças e culturas.

Porém, essa facilidade de acesso a muitas informações pode tornar a busca por um tipo específico de receita ou dieta uma tarefa extremamente complicada. Além disso, a obtenção de informações confiáveis também pode ser muito dificultada, em decorrência da enxurrada de dados à qual os usuários são expostos, sendo, nos dias atuais, cada vez mais utilizadas técnicas avançadas de Aprendizado de Máquina e a Mineração de Dados para a busca de conhecimentos e informações verdadeiramente úteis. Nesse contexto, surgem os Sistemas de Recomendação, que são técnicas de filtragem automática que buscam diminuir significativamente a quantidade de dados resultantes de buscas feitas pelos usuários. Essas técnicas têm sido adaptadas em diversas aplicações dentro do contexto culinário [Nirmal et al. 2018, Gorbonos et al. 2018].

Mesmo com a grande facilidade trazida pelos sistemas de recomendação, a tarefa de busca pela receita desejada ainda é uma tarefa díficil quando há restrições alimentares, como no caso dos alérgicos, veganos, vegetarianos, diabéticos ou alguns grupos religiosos [Ooi et al. 2015]. Repositórios on-line de receitas culinárias dispõem de um número relativamente pequeno de receitas voltadas para as necessidades desses grupos, e que muitas vezes não agradam aos seus usuários. Com isso surgem os Sistemas de Geração de Receitas. Uma das abordagens mais utilizadas para geração de novas receitas, é a substituição de ingredientes em receitas existentes [Oliveira et al. 2019] [Shino et al. 2016] [Ooi et al. 2015]. Essas receitas, por muitas vezes, podem conter ingredientes que são restritos ou proibidos às dietas de certos grupos de usuários. Muitas vezes, a simples substituição de um ingrediente ou de um conjunto reduzido de ingredientes que venham a infringir as restrições de determinados grupos de usuários por ingredientes equivalentes (seja por motivos nutricionais, sabores, texturas, etc.), mas que não façam parte dessas restrições, pode tornar uma receita compatível com as expectativas de tais usuários. Através da substituição de ingredientes, esses usuários podem expandir seu cardápio, adaptando receitas que antes não poderiam consumir.

A ideia é que uma receita imprópria para determinado usuário (receita alvo) se transforme em uma nova receita, que possa ser consumida pelo mesmo, a partir da substituição do ingrediente que causa a inadequação do alimento (ingrediente alvo). Essa geração a partir da substituição de um único ingrediente é realizada no trabalho de [Shino et al. 2016], no qual a sugestão dos ingredientes candidatos (possíveis substitutos do ingrediente alvo) é feita baseada na análise da frequência de coocorrência dos ingredientes na base de receitas. Além disso, é realizada também a categorização dos ingredientes, utilizando para isso ontologias culinárias. 
Contudo, esse tipo substituição é uma difícil tarefa, tendo em vista que vários fatores devem ser levados em consideração, como por exemplo, o papel que determinado ingrediente desempenha na receita e valores nutricionais. Para tentar resolver esse problema, [Ooi et al. 2015] prôpos um método de substituição de ingredientes alergênicos baseado na análise da similaridade de receitas, utilizando dados referentes aos ingredientes, seus estados dentro da receita e modos de preparo, tentando assim capturar o contexto do ingrediente na receita, para achar os substitutos que podem desempenhar o mesmo papel que o ingrediente alvo. Outros fatores também são levados em consideração no trabalho de [Nirmal et al. 2018], no qual um sistema de recomendação é proposto para a geração de receitas pela substituição de um único ingrediente, através de técnicas de otimização tanto do sabor, quanto do valor nutricional dos ingredientes nas recomendações geradas.

Neste trabalho propomos um método de geração de receitas, baseado na substituição de um único ingrediente, que utiliza classificadores lineares para modelar a correlação entre possíveis candidatos a substitutos do ingrediente alvo. Os coeficientes desses classificadores são utilizados para filtrar os candidatos mais correlacionados com a receita, obtendo assim os ingredientes que podem representar os melhores ingredientes substitutos. Esse novo método é proposto como parte de um sistema de geração de receitas para pessoas com dietas especiais, devido a restrições alimentares.

As principais contribuições deste trabalho são:

1. Apresentação e análise do desempenho de um novo método de recomendação de substitutos de ingredientes em receitas culinárias;

2. Desenvolvimento de um sistema de geração de receitas para pessoas com restrições alimentares;

3. Análise da qualidade de dados obtidos em websites, no domínio de receitas culinárias, construídos em conjunto com a comunidade de usuários.

Todas as implementações e execuções de experimentos do presente trabalho foram realizadas pela aluna Larissa Britto, sob orientação da professora Teresa Ludermir e co-orientação do professor Luciano Pacífico, tendo o projeto no qual este trabalho está inserido gerado as seguintes publicações:

- Recipe Recommendation and Generation Based on Ingredient Substitution. Em: Encontro Nacional de Inteligência Artificial e Computacional (ENIAC), 2019 [Oliveira et al. 2019]

- A Text Analysis Approach for Cooking Recipe Classification Based on Brazilian Portuguese Documents. Em: Encontro Nacional de Inteligência Artificial e Computacional, 2019 [Britto et al. 2019a]

- Sistemas de Recomendação de Receitas e Dietas Através de Métodos de Aprendizagem de Máquina. Em: I Encontro de Biociências da UFPE (I EBIO UFPE), 2019 [Britto et al. 2019b]

- Sistemas de Recomendação e Geração de Receitas Através da Categorização Ontológica dos Ingredientes. Em: XII Symposium in Information and Human Language Technology (STIL), 2019 [Pacífico et al. 2019]

O trabalho está organizado da seguinte forma. Na próxima seção (Seção 2), será apresentado o sistema de geração de receitas, incluindo o método de substituição proposto. Uma breve análise da base de dados utilizada e do processamento feito na mesma 
é apresentada na Seção 3. Na Seção 4, os resultados obtidos pelo método proposto são discutidos. Por fim, são apresentadas as conclusões e trabalhos futuros (Seção 5).

\section{Abordagem Proposta}

Nessa seção, propomos um método para a substituição de ingredientes em receitas culinárias, além de um sistema de geração de receitas. As etapas do sistema proposto podem ser vistas na Figura 1. A ideia chave do método proposto é utilizar algoritmos de classificação linear para modelar a correlação entre os ingredientes [Blitzer et al. 2006][Blitzer et al. 2007][Ziser and Reichart 2016][Britto et al. 2019], devido à capacidade desses classificadores de geração de vetores de pesos apropriados para as características de entrada, podendo esses pesos serem então considerados como uma medida de correlação de cada característica com a saída. Através do cálculo da correlação, podemos encontrar ingredientes que são frequentemente utilizados em conjunto e, por tanto, podem ser consideradas boas combinações. Desta forma, utilizando o contexto da receita, podemos então encontrar ingredientes que sejam boas combinações com toda a receita. O classificador escolhido para este trabalho foi a Regressão Logística, um modelo discriminativo utilizado para prever a probabilidade das possíveis saídas de uma variável dependente, dado um conjunto de variáveis independentes [Britto and Pacífico 2019].

O método funciona da seguinte forma: para achar os melhores substitutos do ingrediente alvo $x_{i}$ na receita alvo $R_{t}$, é criado, para cada um dos outros ingredientes em $R_{t}$, um classificador binário que prevê a ocorrência do ingrediente em uma receita qualquer da base de dados. Desta forma, os ingredientes que coocorrem frequentemente com um ingrediente correspondente a determinado classificador, terão uma alta influência na predição desse classificador. Esses classificadores são então treinados com o conjunto $S$ de todas as receitas da base de dados. Como um ingrediente seria altamente preditivo para o seu próprio classificador, todas as ocorrências dos ingredientes da receita alvo são removidas do conjunto de treino.

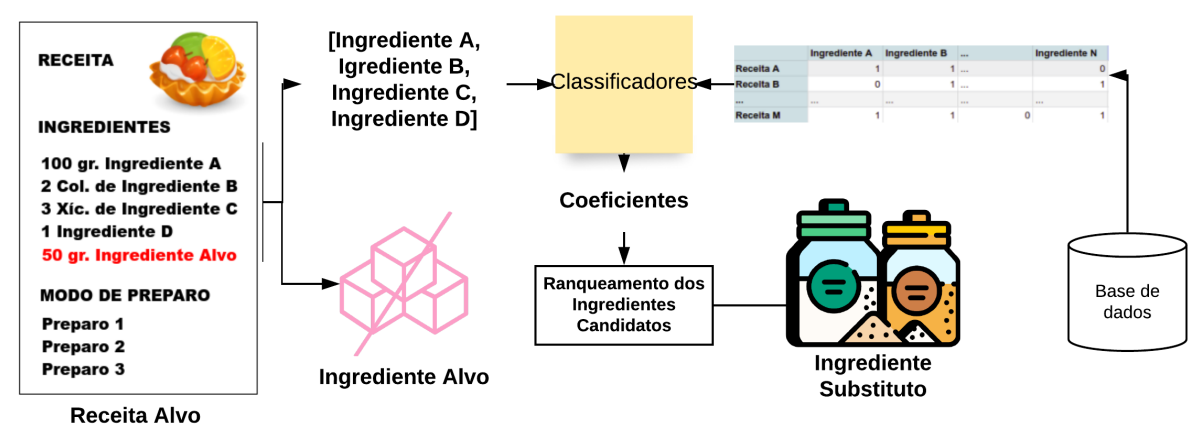

Figura 1. Etapas do Sistema de Geração de Receitas Proposto

Após o treino, os classificadores são representados por seus vetores de coeficientes. O vetor de pesos $\hat{w}$, obtido através da soma dos vetores de coeficientes de todos os classificadores, codificam a covariância dos ingredientes candidatos para os ingredientes da receita. Se o coeficiente assocido a um ingrediente qualquer $x_{z}$ no classificador relativo a um ingrediente $x_{j}$ tem um valor altamente positivo, isso quer dizer que $x_{z}$ e $x_{j}$ são altamente correlacionados e, portanto, representam uma boa combinação. Se esse mesmo ingrediente é altamente correlacionado com todos ou com a maioria dos ingredientes da 
receita alvo, ele poderá ser uma boa opção de substituição ao ingrediente alvo $x_{i}$. Por fim, as melhores recomendações são obtidas através do ranqueamento do vetor de pesos, a partir do qual os ingredientes mais adequados podem ser filtrados. O método proposto leva em consideração o contexto dos alimentos, isto é, um ingrediente pode ter diferentes substitutos, dependendo de sua aplicação e utilidade na receita. O algoritmo completo do método proposto é apresentado no Algoritmo 1.

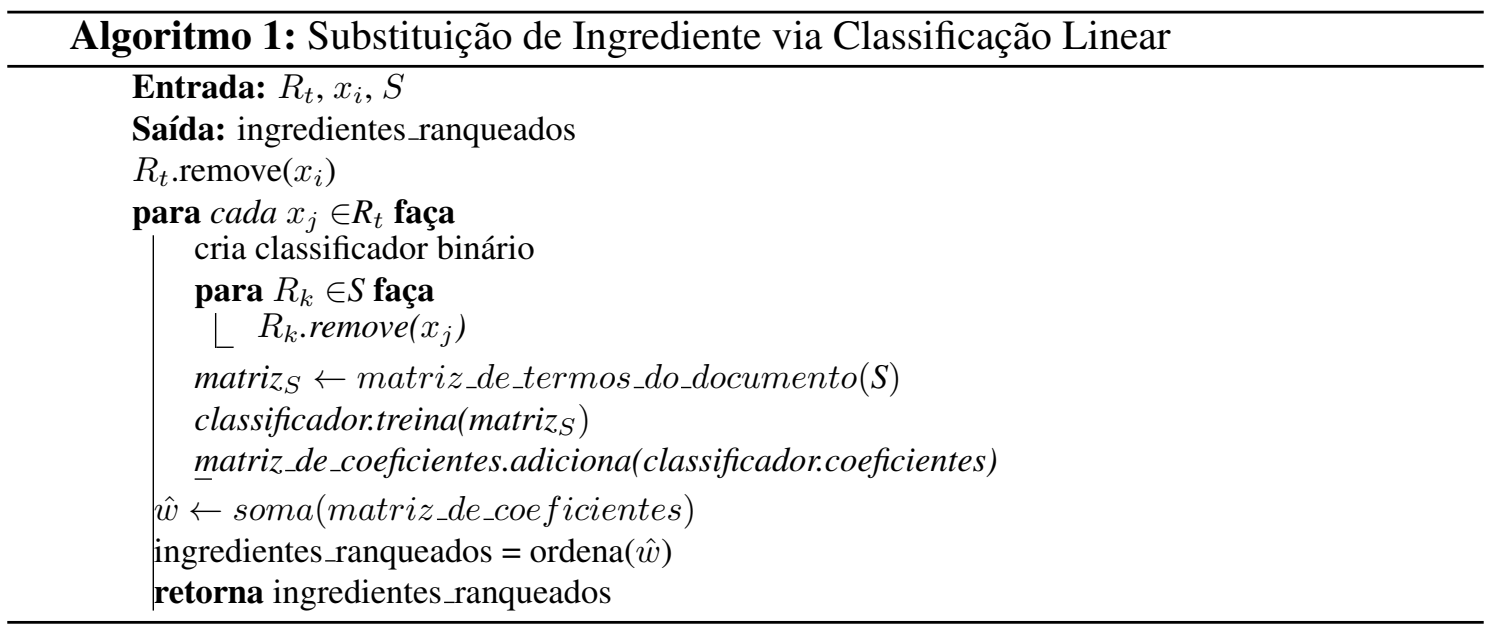

\section{Base de Dados}

A base de dados utilizada neste trabalho foi proposta em [Majumder et al. 2019] e extraída do website Food.com ${ }^{1}$, contendo mais de 230 mil receitas em inglês e 1 milhão de interações de usuários, datadas entre os anos de 2000 e 2018. Dessa base são extraídas as listas de ingredientes de cada receita, além das categorias de restrições e dietas as quais essas receitas se enquadram. Como nosso objetivo é auxiliar usuários com diferentes tipos de restrições alimentares, serão considerados subconjuntos de dados relativos à cada uma dessas restrições, sendo as restrições selecionadas descritas na Tabela 1.

Tabela 1. Informações referentes aos subconjuntos de dados utilizados neste trabalho

\begin{tabular}{|r|c|c|}
\hline Categoria & Diabético & Intolerante à Lactose \\
\hline Descrição & Receitas dedicadas a pessoas diabéticas e a suas restrições & Dieta que não contém lactose \\
\hline Número de Receitas & 6458 & 4105 \\
\hline Total de Ingredientes & 4487 & 3544 \\
\hline Média Ingrediente por Receita & 8.83 & 9.18 \\
\hline Média de Preparos & 8.83 & 9.15 \\
\hline
\end{tabular}

O problema da classificação de receitas culinárias pode ser facilmente mapeado em um problema tradicional de classificacão de textos, no qual a lista total de ingredientes (ou seja, o conjunto formado pela união de todos os ingredientes da base de receitas) é considerado o conjunto final de características do problema [Britto et al. 2019a, Su et al. 2014, Jayaraman et al. 2017, Kalajdziski et al. 2018], e no qual podemos ainda considerar as seguintes relações:

1. Padrão = Documento $=$ Receita;

\footnotetext{
1https://www.kaggle.com/shuyangli 94 /food-com-recipes-and-user-interactions
} 


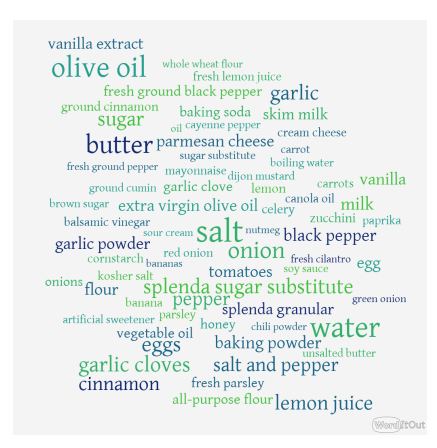

Figura 2. Diabéticos

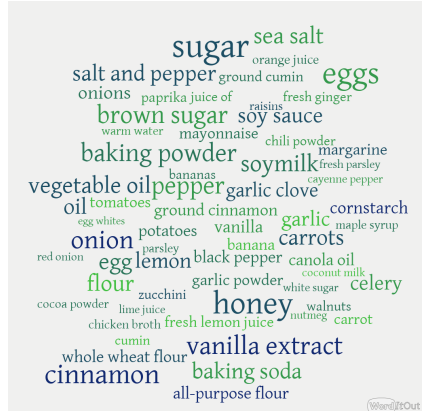

Figura 3. Lactose

2. Característica $=$ Palavra $=$ Ingrediente;

3. Classe $=$ Classe do Documento $=$ Categoria da Receita.

Com base nas relações anteriores, as receitas são então transformadas em uma Matriz de Termos dos Documentos (Document-Term Matrix, ou DTM) [Britto et al. 2019a, Jayaraman et al. 2017, Kalajdziski et al. 2018], para que possam então servir de entrada aos classificadores. Através da DTM foi possível obter os ingredientes mais relevantes em cada subconjunto, que podem ser observados nas Figuras 2 e 3.

\section{Experimentos}

Como forma de avaliaçaõ do método proposto, foram escolhidas aleatóriamente 50 receitas, contendo metade dessas receitas ingredientes impróprios aos diabéticos, e a outra metade, ingredientes impróprios aos intolerantes à lactose. Uma análise qualitativa das substituições obtidas foi realizada através da consulta a sites especializados em culinária e saúde $^{2}$. Além disso, o desempenho do modelo proposto foi avaliado através da análise dos top- $n$ (para $n=1,3,5,10$ ) ingredientes substitutos filtrados para cada uma das receitas, de acordo com três questões de pesquisa:

1. Questão $1(\mathrm{Q} 1)$ : Os ingredientes substitutos gerados são adequados à receita?

2. Questão 2 (Q2): Os ingredientes substitutos gerados são adequados à dieta do usuário e suas restrições?

3. Questão 3 (Q3): Os ingredientes substitutos gerados desempenham o mesmo papel dentro da receita que o ingrediente substituído?

A taxa de substituições para diabéticos e intolerantes à lactose que respondem positivamente às questões de pesquisa levantadas são exibidas nas Figuras 4 e 5 , respectivamente.

Apesar dos subconjuntos de dados de dietas restritivas serem desbalanceados, e isso causar um impacto negativo na performance do método proposto, o mesmo obteve um bom desempenho, tendo apresentado para as top-10 substituições, na maioria dos cenários testados, $100 \%$ de sugestões que continham ao menos um substituto adequado.

O método apresentou um ótimo desempenho para seleção de ingredientes adequados às receitas $(\mathrm{Q} 1)$, até mesmo para o ingrediente top-1, obtendo, nesse caso, uma taxa de acerto de $92 \%$ e $88 \%$ de boas sugestões de substituições para diabéticos e intolerantes à lactose, respectivamente, e $100 \%$ em todos os outros casos (top-3, top-5 e top-10).

\footnotetext{
${ }^{2}$ www. healthline.com, www.medicalnewstoday.com, www.bhg.com
} 


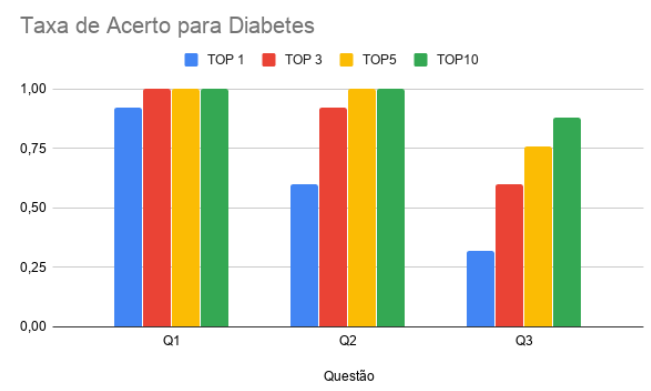

Figura 4. Taxa de Acerto nas Substituições para Diabéticos

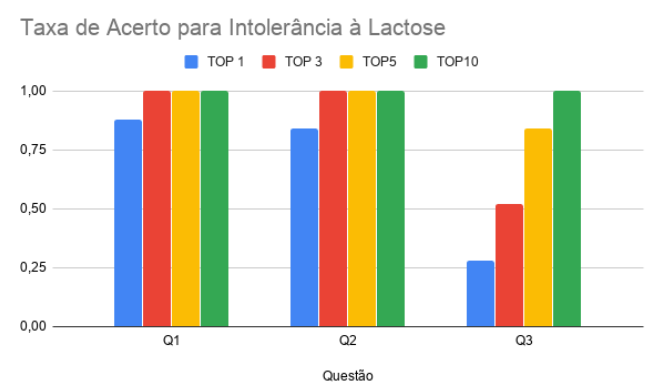

Figura 5. Taxa de Acerto nas Substituições para Intolerantes à Lactose

A substituição por ingredientes que se adequem à restrição dos usuários $(\mathrm{Q} 2)$ foi impactada negativamente pela qualidade da base de dados, dado que receitas inadequadas a determinadas categorias de dietas foram errôneamente inseridas na base de dados pelos usuários (ou seja, categorizações incorretas foram fornecidas à base de dados). Mesmo com esse impacto, o método proposto ainda foi capaz de aprasentar um bom desempenho, tendo resultados aceitáveis sido obtidos a partir das top-3 substituições, com $92 \%$ de acerto nas substituições para diabéticos, e $100 \%$ de sugestões de boas substituições para todos os demais casos (top-3 para intolerância à lactose, assim como no top-5 e top-10 para ambos os casos).

A tarefa mais complexa para o sistema automático de substituição de ingredientes proposto é, sem dúvidas, a obtenção de ingredientes que fossem capazes de desempenhar o mesmo papel que o ingrediente substituído possuía na receita original (Q3). O método foi capaz de obter bons resultados para essa tarefa a partir dos top-5 substitutos, alcançando uma taxa de acerto de $76 \%$ e $84 \%$ nas substituições nessa avaliação para diabéticos e intolerantes à lactose, respectivamente, e taxas de boas recomendações de $88 \%$ e $100 \%$, para os top-10 substituitos em receitas para diabéticos e intolerantes à lactose, respectivamente. Esse resultado demonstra o bom desempenho do método proposto, principalmente levando em consideração que os ingredientes substituídos adotados nos experimentos podem ser utilizados em receitas de diversas categorias, o que faz com que categorias de receitas dominantes influenciem negativamente nas categorias com menor número de receitas.

Para uma melhor avaliação do método proposto, foram escolhidas aleatoriamente duas receitas, tanto para exemplificar seu uso, quanto para a realização de uma avaliação qualitativa do mesmo. As receitas escolhidas são apresentadas na Tabela 2. Os resultados 
da aplicação do método proposto às receitas alvo (Banana Sour Cream Crunch Bake e Caramel Apple Upside-down Cornmeal Cake) podem ser observados na Tabela 3. Os resultados mostram a capacidade do método de filtragem proposto de oferecer boas soluções, tendo o mesmo obtido, para todos os casos avaliados, mais de uma recomendação de ingrediente substituto de qualidade, sendo apresentados ingredientes que se encaixavam e supriam o dever do ingrediente original dentro da receita, e ainda se mostravam uma melhor alternativa para a restrição do usuário.

Tabela 2. Lista de Ingredientes das Receitas Alvo (em Inglês)

\begin{tabular}{|l|l|}
\hline Receita & Ingredientes \\
\hline Banana Sour Cream Crunch Bake & $\begin{array}{l}\text { butter, sugar, eggs, mashed banana, vanilla, sour cream, } \\
\text { flour, baking, powder, baking soda, salt, butterscotch } \\
\text { chips, ground cinnamon }\end{array}$ \\
\hline $\begin{array}{l}\text { Caramel Apple Upside-down Corn- } \\
\text { meal Cake }\end{array}$ & $\begin{array}{l}\text { butter, apples, brown sugar, milk, pecans, dried cranber- } \\
\text { ries, flour, sugar, baking powder, salt, cornmeal, hot wa- } \\
\text { ter, eggs, vanilla }\end{array}$ \\
\hline
\end{tabular}

Apesar da maioria dos substitutos serem adequados dentro da receita e para o usuário, algumas sugestões obtidas foram inesperadas, tendo em vista que não estavam dentro dos alimentos que podiam ser consumidos pelo usuário devido às suas restrições. Isso se deve ao fato de que o website de onde a base de dados foi extraída permite a qualquer usuário a inserção de receitas. Apesar dessa abordagem ter pontos positivos, como a grande variabilidade e volume de receitas, a mesma também apresenta várias desvantagens, como a apresentação de informações e categorizações incorretas ao sistema por parte dos usuários. Algumas receitas para intolerantes à lactose, por exemplo, eram compostas por ingredientes como leite. A má qualidade dos dados não impactou significativamente o desempenho do método proposto, porém levanta atenção para que os dados armazenados em tais sistemas, dada a importâncias dos mesmos, sejam sempre desenvolvidos e avaliados por especialistas.

\section{Conclusões}

Neste trabalho foi proposto um método de filtragem de ingredientes para substituição em receitas culinárias, através do qual receitas podem ser adaptadas para atender restrições e gostos pessoais dos usuários. A abordagem proposta, que utiliza classificadores lineares para modelar a correlação entre os ingredientes candidatos à substituição e os ingredientes da receita, apresentou um bom desempenho, tendo tal modelo obtido substitutos adequados na grande maioria dos cenários investigados, apesar da existência de problemas na base de dados utilizada, como o desbalanceamento entre a quantidade de receitas nas categorias, e a presença de categorizações incorretas, decorrentes de erros de rotulação cometidos pelos usuários. Uma análise qualitativa demonstrou a importância da qualidade dos dados e da existência de um especialista para que informações errôneas não comprometam o desempenho do sistema.

Como trabalhos futuros, pretedemos avaliar o desempenho de outros classificadores, como as Máquinas de Vetores de Suporte e as Redes Neurais Artificiais, para analisar qual seria a melhor opção para compor o módulo de substituição de ingredientes e geração de receitas do sistema. Além disso, pretedemos utilizar outros tipos de restrições 
Tabela 3. Resultados dos Top-10 Ingredientes Candidatos a substituição. Em negrito os ingredientes que são adequados para a substituição.

\begin{tabular}{|c|c|c|c|c|c|}
\hline \multicolumn{2}{|c|}{ Receita } & \multicolumn{2}{|c|}{ Banana Sour Cream Crunch Bake } & \multicolumn{2}{|c|}{ Caramel Apple Upside-down Cornmeal Cake } \\
\hline Ingrediente Original & Restrição & Ingrediente Candidato & Peso & Ingrediente Candidato & Peso \\
\hline \multirow{10}{*}{ butter } & \multirow{10}{*}{ Intolerância à Lactose } & nuts & 7.3885 & baking soda & 9.2944 \\
\hline & & oil & 6.9562 & cinnamon & 8.4826 \\
\hline & & shortening & 6.5126 & oil & 6.9711 \\
\hline & & applesauce & 6.3307 & ground cinnamon & 6.4257 \\
\hline & & canola oil & 6.2595 & nuts & 5.8241 \\
\hline & & unsweetened applesauce & 6.2146 & margarine & 5.5050 \\
\hline & & ground cloves & 5.7815 & vegetable oil & 5.2074 \\
\hline & & cornmeal & 5.3781 & shortening & 5.053 \\
\hline & & cocoa powder & 5.3753 & orange juice & 4.9628 \\
\hline & & margarine & 5.3270 & unsalted margarine & 4.7673 \\
\hline \multirow{10}{*}{ sugar } & \multirow{10}{*}{ Diabético } & dark brown sugar & 9.3243 & coconut & 8.0663 \\
\hline & & powdered sugar & 8.7773 & shortening & 7.8344 \\
\hline & & buttermilk & 8.4706 & dark brown sugar & 7.0535 \\
\hline & & xylitol sweetener & 7.1117 & xylitol sweetener & 6.3634 \\
\hline & & almond flour & 6.5725 & baking soda & 6.3510 \\
\hline & & shortening & 6.4846 & powdered sugar & 6.0873 \\
\hline & & splenda granular & 6.0515 & powdered sugar & 6.0873 \\
\hline & & applesauce & 5.8670 & self-rising flour & 5.4308 \\
\hline & & egg & 5.8554 & light margarine & 5.2372 \\
\hline & & stevia & 5.8076 & stevia & 5.0013 \\
\hline
\end{tabular}

para analisar como os diferentes domínios e receitas de diferentes complexidades reagem ao método proposto. Pretendemos ainda disponibilizar para o público um sistema completo de recomendação e geração de receitas culinárias, que leve em consideração as necessidades e desejos de seus usuários.

\section{Referências}

Blitzer, J., Dredze, M., and Pereira, F. (2007). Biographies, bollywood, boom-boxes and blenders: Domain adaptation for sentiment classification.

Blitzer, J., McDonald, R., and Pereira, F. (2006). Domain adaptation with structural correspondence learning. In Proceedings of the 2006 Conference on Empirical Methods in Natural Language Processing, pages 120-128, Sydney, Australia. Association for Computational Linguistics.

Britto, L. F. S., Lima, R., and Pacífico, L. D. S. (2019). Structural correspondence learning for cross-domain sentiment analysis in brazilian portuguese. In 2019 8th Brazilian Conference on Intelligent Systems (BRACIS), pages 812-817.

Britto, L. F. S., Oliveira, E. G., Pacífico, L. D. S., and Ludermir, T. B. (2019a). Uma abordagem de análise de textos para a classificação de receitas culinárias baseadas em documentos em português brasileiro. In Anais do XVI Encontro Nacional de Inteligência Artificial e Computacional, pages 436-447, Porto Alegre, RS, Brasil. SBC.

Britto, L. F. S. and Pacífico, L. D. S. (2019). Análise de sentimentos para revisões de aplicativos mobile em português brasileiro. In Anais do XVI Encontro Nacional de Inteligência Artificial e Computacional, pages 1080-1090, Porto Alegre, RS, Brasil. SBC.

Britto, L. F. S., Pacífico, L. D. S., and Ludermir, T. B. (2019b). Sistemas de recomendação de receitas e dietas através de métodos de aprendizagem de máquina. In Anais do I Encontro de Biociências da UFPE (I EBIO UFPE). 
Gorbonos, E., Liu, Y., and Hoàng, C. T. (2018). Nutrec: Nutrition oriented online recipe recommender. In 2018 IEEE/WIC/ACM International Conference on Web Intelligence (WI), pages 25-32. IEEE.

Jayaraman, S., Choudhury, T., and Kumar, P. (2017). Analysis of classification models based on cuisine prediction using machine learning. In 2017 International Conference On Smart Technologies For Smart Nation (SmartTechCon), pages 1485-1490. IEEE.

Kalajdziski, S., Radevski, G., Ivanoska, I., Trivodaliev, K., and Stojkoska, B. R. (2018). Cuisine classification using recipe's ingredients. In 201841 st International Convention on Information and Communication Technology, Electronics and Microelectronics (MIPRO), pages 1074-1079. IEEE.

Majumder, B. P., Li, S., Ni, J., and McAuley, J. (2019). Generating personalized recipes from historical user preferences. In Proceedings of the 2019 Conference on Empirical Methods in Natural Language Processing and the 9th International Joint Conference on Natural Language Processing (EMNLP-IJCNLP), pages 5976-5982, Hong Kong, China. Association for Computational Linguistics.

Nirmal, I., Caldera, A., and Bandara, R. D. (2018). Optimization framework for flavour and nutrition balanced recipe: A data driven approach. In 2018 5th IEEE Uttar Pradesh Section International Conference on Electrical, Electronics and Computer Engineering (UPCON), pages 1-9. IEEE.

Oliveira, E. G., Britto, L. F. S., Pacífico, L. D. S., and Ludermir, T. B. (2019). Recomendação e geração de receitas baseada na substituição de ingredientes. In Anais do XVI Encontro Nacional de Inteligência Artificial e Computacional, pages 238-249, Porto Alegre, RS, Brasil. SBC.

Ooi, A., Iiba, T., and Takano, K. (2015). Ingredient substitute recommendation for allergy-safe cooking based on food context. In 2015 IEEE Pacific Rim Conference on Communications, Computers and Signal Processing (PACRIM), pages 444-449. IEEE.

Pacífico, L. S. D., Oliveira, E. G., Britto, L. F. S., and Ludermir, T. B. (2019). Sistemas de recomendação e geração de receitas através da categorizacão ontológica dos ingredientes. In Proceedings of the XII Brazilian Symposium in Information and Human Language Technology, pages 80-85.

Shino, N., Yamanishi, R., and Fukumoto, J. (2016). Recommendation system for alternative-ingredients based on co-occurrence relation on recipe database and the ingredient category. In 2016 5th IIAI International Congress on Advanced Applied Informatics (IIAI-AAI), pages 173-178. IEEE.

Su, H., Lin, T.-W., Li, C.-T., Shan, M.-K., and Chang, J. (2014). Automatic recipe cuisine classification by ingredients. In Proceedings of the 2014 ACM international joint conference on pervasive and ubiquitous computing: adjunct publication, pages 565-570. ACM.

Ziser, Y. and Reichart, R. (2016). Neural structural correspondence learning for domain adaptation. CoRR, abs/1610.01588. 